\title{
Abundance gradients in the Milky Way for $\alpha$ elements, iron peak elements, barium, lanthanum, and europium ${ }^{\star}$
}

\author{
G. Cescutti ${ }^{1}$, F. Matteucci ${ }^{1,2}$, P. François ${ }^{3,4}$, and C. Chiappini ${ }^{2}$ \\ 1 Dipartimento di Astronomia, Universitá di Trieste, via G.B. Tiepolo 11, 34131 Trieste, Italy \\ e-mail: cescutti@oats.inaf.it \\ 2 I.N.A.F. Osservatorio Astronomico di Trieste, via G.B. Tiepolo 11, 34131 Trieste, Italy \\ 3 Observatoire de Paris/Meudon, GEPI, 61 avenue de l'Observatoire, 75014 Paris, France \\ ${ }^{4}$ European Southern Observatory, Casilla 19001, Santiago 19, Chile
}

Received 10 April 2006 / Accepted 25 September 2006

ABSTRACT

\begin{abstract}
We model the abundance gradients in the disk of the Milky Way for several chemical elements $(\mathrm{O}, \mathrm{Mg}, \mathrm{Si}, \mathrm{S}, \mathrm{Ca}, \mathrm{Sc}, \mathrm{Ti}, \mathrm{Co}, \mathrm{V}$, $\mathrm{Fe}, \mathrm{Ni}, \mathrm{Zn}, \mathrm{Cu}, \mathrm{Mn}, \mathrm{Cr}, \mathrm{Ba}, \mathrm{La}$ and $\mathrm{Eu}$ ), and compare our results with the most recent and homogeneous observational data. We adopt a chemical evolution model able to well reproduce the main properties of the solar vicinity. The model assumes that the disk formed inside-out with a timescale for the formation of the thin disk of $7 \mathrm{Gyr}$ in the solar vicinity, whereas the halo formed on a timescale of $0.8 \mathrm{Gyr}$. We adopt new empirical stellar yields derived to best fit the abundances and the abundance ratios in the solar vicinity. We compute, for the first time, the abundance gradients for all the mentioned elements in the galactocentric distance range 4-22 kpc. Comparison with the observed data on Cepheids in the galactocentric distance range 5-17 kpc gives a very good agreement for many of the studied elements. In addition, we well fit the data for the evolution of lanthanum in the solar vicinity, for which we present results here for the first time. We explore, also for the first time, the behavior of the abundance gradients at large galactocentric distances by comparing our results with data relative to distant open clusters and red giants and select the best chemical evolution model on that basis. We find a very good fit to the observed abundance gradients, as traced by Cepheids, for most of the elements, thus confirming the validity of the inside-out scenario for the formation of the Milky Way disk as well as the adopted nucleosynthesis prescriptions. The flat gradients at large galactocentric distances $(>12 \mathrm{kpc})$, as traced by the Cepheids, open cluster and red giant data, lead us to conclude that a model where the density of the halo stellar component is constant in the inner $20 \mathrm{kpc}$ should be preferred. Other models with different distributions of the halo stellar mass do not produce a good fit of the data.
\end{abstract}

Key words. nuclear reactions, nucleosynthesis, abundances - Galaxy: abundances - Galaxy: evolution

\section{Introduction}

Understanding the formation and the evolution of the Milky Way is fundamental to improve the knowledge of the formation of spiral galaxies in general. There are many observational constraints in the Milky Way. The most important ones are represented by the evolution of the abundances of the chemical elements. Recently, many chemical evolution models have been developed to explain the chemical composition of the solar vicinity and the $[\mathrm{el} / \mathrm{Fe}]$ vs. $[\mathrm{Fe} / \mathrm{H}]$ patterns (e.g. Henry et al. 2000; Liang et al. 2001; Chiappini et al. 2003b, 2003a; Akerman et al. 2004; François et al. 2004). Other important constraints, which are connected to the evolution of the Galaxy disk, are the abundance gradients of the elements along the disk of the Milky Way. Abundance gradients are a feature commonly observed in many galaxies with their metallicities decreasing outward from the galactic centers. The study of the gradients provides strong constraints to the mechanism of galaxy formation; in fact, star formation and the accretion history as functions of galactocentric distance in the galactic disk strongly influence the formation and the development of the abundance gradients (see Matteucci \& François 1989; Boisser \& Prantzos 1999; Chiappini et al. 2001). Many models have been computed to explain the behaviour of

* Full Table 4 is only available in electronic form at http: //www . aanda.org abundances and abundance ratios as functions of galactocentric radius (e.g. Hou et al. 2000; Chang et al. 1999; Chiappini et al. 2003a; Alibés et al. 2001) but they restrict their predictions to a small number of chemical elements and do not consider very heavy elements.

We base our work on the chemical evolution model described in Chiappini et al. (2001), which is able to well reproduce the gradients in the Milky Way for N, O, S and Fe.

In this work we calculate the behavior of the largest number of heavy elements $(\mathrm{O}, \mathrm{Mg}, \mathrm{Si}, \mathrm{S}, \mathrm{Ca}, \mathrm{Sc}, \mathrm{Ti}, \mathrm{Co}, \mathrm{V}, \mathrm{Fe}, \mathrm{Ni}$, $\mathrm{Zn}, \mathrm{Cu}, \mathrm{Mn}, \mathrm{Cr}, \mathrm{Ba}, \mathrm{La}$ and $\mathrm{Eu}$ ) ever considered in this kind of model. We are also able to test the the recent nucleosynthesis prescriptions described in François et al. (2004) for the $\alpha$ and iron peak elements and in Cescutti et al. (2006) for Eu and Ba; the prescriptions for lanthanum are newly calculated in this paper following the same approach adopted for barium in the paper by Cescutti et al. (2006).

Chemical evolution models adopting the above nucleosynthesis prescriptions have been shown to reproduce the evolution of the abundances in the solar neighborhood. Here we extend our predictions to the whole disk to check whether these models also can reproduce the abundance gradients. We compare our model predictions to new observational data collected by Andrievsky et al. (2002a-c, 2004) and Luck et al. (2003, hereafter 4AL). They measured the abundances of all the selected 
elements (except $\mathrm{Ba}$ ) in a sample of 130 galactic Cepheids found in the galactocentric distance range from 5 to $17 \mathrm{kpc}$. In addition to the data by $4 \mathrm{AL}$ we compare our theoretical predictions with abundance measurements in giants and open clusters located at even larger galactocentric distances.

The paper is organized as follows: in Sect. 2 we present the observational data, in Sect. 3 the chemical evolution model is presented and in Sect. 4 the adopted nucleosynthesis prescriptions are described. In Sect. 5 we present the results and in Section 6 some conclusions are drawn.

\section{Observational data}

In this work we use the data by $4 \mathrm{AL}$ for all the studied elemental gradients. These accurate data have been derived for a large sample of galactic Cepheids. Cepheids variables have a distinct role in the determination of radial abundance gradients for a number of reasons. First, they are usually bright enough that they can be observed at large distances, providing accurate abundances; second, their distances are generally well determined, as these objects are often used as distance calibrators (see Feast \& Walker 1987); third, their ages are also well determined, on the basis of relations involving their periods, luminosities, masses and ages (see Bono et al. 2005). They generally have ages close to a few hundred million years. We can thus safely assume that they are representative of the present day gradients. The 4AL sample contains abundance measurements for 130 Cepheid stars located between 5 and $17 \mathrm{kpc}$ from the Galactic center, for all the elements we want to study but $\mathrm{Ba}$. The advantage of this data is that it constitutes a homogeneous sample for a large number of stars and measured elements. Therefore, the abundance gradients can be more closely traced with better statistics. Moreover, only for Cepheids it is possible to obtain abundances for so many elements, as well as a good estimate of the distance, necessary to compute the gradients. 4AL obtained multiphase observations for the majority of the stars. For the distant Cepheids they used 3-4 spectra to derive the abundances, while for nearby stars 2-3 spectra were used. We also adopt the data by Yong et al. (2006), who computed the chemical abundances of $\mathrm{Fe}, \mathrm{Mg}, \mathrm{Si}$, $\mathrm{Ca}, \mathrm{Ti}, \mathrm{La}$ and Eu for 30 Cepheids stars. Among these 30 stars, we choose only the 20 which are not in common with the sample of 4AL. We apply the off-set found by Yong et al. (2006) with respect to the work of $4 \mathrm{AL}$, in order to homogenize the two samples.

We use the data of Daflon \& Cuhna (2004) to compare the results on Cepheids with another class of young objects. Their database contains abundances of $\mathrm{C}, \mathrm{N}, \mathrm{O}, \mathrm{Mg}, \mathrm{Al}, \mathrm{Si}, \mathrm{S}$ for 69 late $\mathrm{O}$ - to early B-type star members of $25 \mathrm{OB}$ associations, open clusters and HII regions. They determine the mean abundances of the different clusters or associations of young objects. These objects all have ages less than 50 Myr. Therefore, we assume that they also represent the present day gradients.

To extend the comparison between our model and the observational data toward the outer disk, we also include the datasets of Carraro et al. (2004) and Yong et al. (2005); these authors observed distant open clusters up to $22 \mathrm{kpc}$. We also show the average values of individual stars belonging to a cluster. These stars are red giants with an estimated age ranging from 2 Gyr to 5 Gyr. We compare these data with the results of our model at the sun formation epoch, i.e. $4.5 \mathrm{Gyr}$ before the present time. Yong et al. (2005) measured the surface abundances of $\mathrm{O}$, $\mathrm{Mg}, \mathrm{Si}, \mathrm{Ca}, \mathrm{Ti}, \mathrm{Mn}, \mathrm{Co}, \mathrm{Ni}, \mathrm{Fe} \mathrm{La}, \mathrm{Eu}$ and $\mathrm{Ba}$ for 5 clusters, whereas Carraro et al. (2004) computed the surface abundances of $\mathrm{O}, \mathrm{Mg}, \mathrm{Si}, \mathrm{Ca}, \mathrm{Ti}, \mathrm{Ni}, \mathrm{Fe}$ in 2 clusters. One of the clusters of
Carraro et al. (2004), Berkeley 29, is in common with the sample of Yong et al. (2005) and we show both measurements. The galactocentric distance of this object is $22 \mathrm{kpc}$ and hence is the most distant open cluster ever observed.

We show the abundances of three field red giants, which have been identified in the direction of the southern warp of the Galaxy by Carney et al. (2005). In their work, they measure the abundances of $\mathrm{O}, \mathrm{Mg}, \mathrm{Si}, \mathrm{Ca}, \mathrm{Ti}, \mathrm{Mn}, \mathrm{Co}, \mathrm{Ni}, \mathrm{Fe}, \mathrm{La}, \mathrm{Eu}$ and $\mathrm{Ba}$ for the three red giants. The galactocentric distance of these object ranges from $10 \mathrm{kpc}$ to $15 \mathrm{kpc}$. The age of these three stars is unknown but it is likely that it is similar to the age of the red giants measured in the old open clusters. Therefore, we may compare them with the abundances at the solar system formation time.

\section{The chemical evolution model for the Milky Way}

In our model, the Galaxy is assumed to have formed by means of two main infall episodes: the first forms the halo and the thick disk, the second the thin disk. The timescale for the formation of the halo-thick disk is 0.8 Gyr. The timescale for the thin disk is much longer, 7 Gyr in the solar vicinity, implying that the infalling gas forming the thin disk comes mainly from the intergalactic medium and not only from the halo (Chiappini et al. 1997). Moreover, the formation of the thin disk is assumed to be a function of the galactocentric distance, leading to an inside out scenario for the Galaxy disk build-up (Matteucci \& François 1989). The galactic thin disk is approximated by several independent rings, $2 \mathrm{kpc}$ wide, without exchange of matter between them.

The main characteristic of the two-infall model is an almost independent evolution between the halo and the thin disk (see also Pagel \& Tautvaisiene 1995). A threshold gas density of $7 M_{\odot} \mathrm{pc}^{-2}$ in the star formation process (Kennicutt 1989, 1998; Martin \& Kennicutt 2001) is also adopted for the disk.

The model well reproduces an extended set of observational constraints, in particular for the solar neighborhood. Some of the most important observational constraints are represented by the various relations between the abundances of metals $(\mathrm{C}, \mathrm{N}, \mathrm{O}, \alpha$ elements, iron peak elements) as functions of the $[\mathrm{Fe} / \mathrm{H}]$ abundance (see Chiappini et al. 2003a,b; François et al. 2004) and by the G-dwarf metallicity distribution. Although this model is probably not unique, it reproduces the majority of the observed features of the Milky Way. Many of the assumptions of the model are shared by other authors (e.g. Prantzos \& Boissier 2000; Alibés et al. 2001; Chang et al. 1999).

Chiappini et al. (2001) have shown that the chemical evolution of the halo can have an impact on the abundance gradients in the outer parts of the disk. They analyzed the influence of the halo surface mass density on the formation of the abundance gradients of $\mathrm{O}, \mathrm{S}, \mathrm{Fe}$ and $\mathrm{N}$ at large galactocentric distances. In their model A, the halo surface mass density is assumed constant and equal to $17 M_{\odot} \mathrm{pc}^{-2}$ for $R \leq 8 \mathrm{kpc}$ and decreases as $R^{-1}$ outward. A threshold of gas density is assumed also for the halo and set to $4 M_{\odot} \mathrm{pc}^{-2}$. Then model B has a constant surface mass density equal to $17 M_{\odot} \mathrm{pc}^{-2}$ for all galactocentric distances and the threshold in the halo phase is the same as in model A. In their model $\mathrm{C}$ the halo surface mass density is assumed as in model A but it does not have a threshold in the halo phase. In their model D, both the halo surface mass density and the threshold are as in model A but the time scale for the halo formation at galactocentric distances greater than $10 \mathrm{kpc}$ is set to $2 \mathrm{Gyr}$ and to $0.8 \mathrm{Gyr}$ for distances less than $10 \mathrm{kpc}$. In all the other models, the halo timescale is constant for all the galactocentric 
distances and equal to 0.8 Gyr. Here we will show our model predictions for model $\mathrm{B}$. The differences among model A, B, C and $\mathrm{D}$ arise primarily in the predicted steepness of the gradients for the outermost disc regions of the galactic disc. In this zone, the model B predicts the flattest gradients among the models of Chiappini et al. (2001), and provides the best fit according to observed flatness in the recent data by $4 \mathrm{AL}$ and in the distant open clusters. Model A is also in good agreement with the abundance gradients traced by Cepheids up to $\sim 12 \mathrm{kpc}$, whereas for larger galactocentric distances this model tends to be systematically below observations. We will show the predictions of this model only for the $\alpha$-elements. Model C shows a trend similar to model A, whereas model D tends to be below the observations for galactocentric distances greater than $10 \mathrm{kpc}$, so we chose to not show their predictions. We do not give a detailed description of the model, which can be found in Chiappini et al. (2001); nevertheless, to better understand how the gradients form, it is fundamental to know how we model the built up of the disk and the halo, and thus the rate of mass accretion $A(r, t)$, which is a function of time and galactocentric distance:

$A(r, t)=a(r) \mathrm{e}^{-t / \tau_{\mathrm{H}}}+b(r) \mathrm{e}^{\left(t-t_{\max }\right) / \tau_{\mathrm{D}}(r)}$.

In this equation, $t_{\max }=1 \mathrm{Gyr}$ is the time for the maximum infall rate on the thin disk, $\tau_{\mathrm{H}}=0.8 \mathrm{Gyr}$ is the time scale for the formation of the halo thick-disk and $\tau_{\mathrm{D}}$ is the timescale of the thin disk, which is a function of the galactocentric distance:

$\tau_{\mathrm{D}}=1.033 r(\mathrm{kpc})-1.267 \mathrm{Gyr}$.

The coefficients $a(r)$ and $b(r)$ are constrained to reproduce the present day total surface mass density as a function of galactocentric distance. In particular, $b(r)$ is assumed to be different from zero only for $t>t_{\max }$, where $t_{\max }$ is the time of maximum infall on the thin disk (see Chiappini et al. 2003b, for details). Another important ingredient of the model is the adopted law for the SFR, which is the following:

$\psi(r, t)=v\left(\frac{\Sigma(r, t)}{\Sigma\left(r_{\odot}, t\right)}\right)^{2(k-1)}\left(\frac{\Sigma\left(r, t_{\mathrm{Gal}}\right)}{\Sigma(r, t)}\right)^{k-1} G_{\mathrm{gas}}^{k}(r, t)$.

$v$ is the efficiency of the star formation process and is set to be $2 \mathrm{Gyr}^{-1}$ for the Galactic halo $\left(t<1 \mathrm{Gyr}\right.$ ) and $1 \mathrm{Gyr}^{-1}$ for the disk ( $t \geq 1 \mathrm{Gyr}) . \Sigma(r, t)$ is the total surface mass density, $\Sigma\left(r_{\odot}, t\right)$ the total surface mass density at the solar position, $G_{\mathrm{gas}}(r, t)$ the surface density normalized to the present time total surface mass density in the disk $\Sigma_{\mathrm{D}}\left(r, t_{\mathrm{Gal}}\right)$, where $t_{\mathrm{Gal}}=13.7 \mathrm{Gyr}$ is the age assumed for the Milky Way and $r_{\odot}=8 \mathrm{kpc}$ the solar galactocentric distance (Reid 1993). The exponent of the surface gas density, $k$, is set equal to 1.5 . With these values for the parameters the observational constraints, in particular in the solar vicinity, are well fitted. We recall that below a critical threshold for the gas surface density $\left(7 M_{\odot} \mathrm{pc}^{-2}\right.$ for the thin disk and $4 M_{\odot} \mathrm{pc}^{-2}$ for the halo phase) we assume no star formation.

In Fig. 1 we show the predicted star formation rate for three different galactocentric distances: 4,8 and $12 \mathrm{kpc}$; the SFR is the same for all galactocentric distances during the halo phase, due to the fact that the assumed halo mass density in the selected model B is not a function of galactocentric distance; the critical threshold of the gas surface density naturally produces a bursting star formation history in the outer part of the disk, whereas at the solar neighborhood, it happens only toward the end of the evolution. We note that at the solar galactocentric distance, which is assumed to be $8 \mathrm{kpc}$, the threshold also produces a hiatus between the halo phase and the thin disk phase.

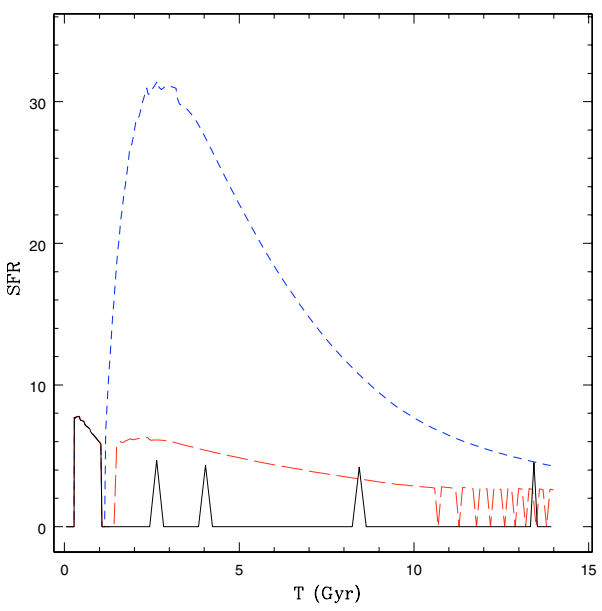

Fig. 1. The SFR expressed in $M_{\odot} \mathrm{pc}^{-2} \mathrm{Gyr}^{-1}$, as predicted by the two infall model for different galactocentric distances: $4 \mathrm{kpc}$ (short dashed line), $8 \mathrm{kpc}$ (long dashed line) and $12 \mathrm{kpc}$ (solid line). The SFR in the halo phase (indicated by the solid line) up to $0.8 \mathrm{Gyr}$, is the same for all the galactocentric distances, whereas in the disk the SFR changes according to the different infall rates. Note that at $4 \mathrm{kpc}$ distance the SFR in the disk is much higher than at larger galactocentric distances. The gap in the SFR at the end of the halo-thick disk phase is evident in the solar neighborhood. The oscillations are due to to the threshold density.

\section{Nucleosynthesis prescriptions}

\section{1. $\alpha$ and iron peak elements}

For the nucleosynthesis prescriptions of the Fe and the others elements (O, S, Si, Ca, Mg, Sc, Ti, V, Cr, Zn, Cu, Ni, Co and Mn), we adopted those suggested in François et al. (2004). They compared theoretical predictions of the $[\mathrm{el} / \mathrm{Fe}]$ vs. $[\mathrm{Fe} / \mathrm{H}]$ trends in the solar neighborhood for the mentioned above elements and they selected the best sets of yields to fit the data. For the yields of SNe II they found that the Woosley \& Weaver (1995) ones provide the best fit. No modifications are required for the yields of $\mathrm{Ca}, \mathrm{Fe}, \mathrm{Zn}$ and $\mathrm{Ni}$ as computed for solar composition. For oxygen the best results are given by the Woosley \& Weaver (1995) yields computed as functions of the metallicity. For the other elements, variations in the predicted yields are required to best fit the data (see François et al. 2004, for details). For yields from type SNeIa, revisions in the theoretical yields by Iwamoto et al. (1997) are needed for $\mathrm{Mg}, \mathrm{Ti}, \mathrm{Sc}, \mathrm{K}, \mathrm{Co}, \mathrm{Ni}$ and $\mathrm{Zn}$ to best fit the data. The prescription for single low-intermediate mass stars are by van den Hoek \& Groenewegen (1997), for the case of the mass loss parameter which varies with metallicity (see Chiappini et al. 2003b, model 5).

\section{2. $S$ and $R$ process}

For the nucleosynthesis prescriptions of $s$-process we have adopted the yields of Busso et al. (2001) in the mass range 1.5-3 $M_{\odot}$ for lanthanum and barium. The theoretical results by Busso et al. (2001) suggest negligible Europium production in the $s$-process and therefore we neglected this component in our work. We extended the theoretical results of Busso et al. (2001) in the mass range $1.5-1 M_{\odot}$, by simply scaling the values obtained for stars of $1.5 M_{\odot}$ by the mass. We extended the prescriptions to better fit the data with a $[\mathrm{Fe} / \mathrm{H}]$ higher than solar. This hypothesis does not change the results of the model at $[\mathrm{Fe} / \mathrm{H}]<0$. 
Table 1. The stellar yields for La in massive stars ( $r$-process) in the case of primary origin.

\begin{tabular}{cc}
\hline \hline$M_{\text {star }}$ & $X_{\mathrm{La}}^{\text {new }}$ \\
\hline 12. & $9.00 \times 10^{-8}$ \\
15. & $3.00 \times 10^{-9}$ \\
30. & $1.00 \times 10^{-10}$ \\
\hline
\end{tabular}

For the nucleosynthesis prescriptions of $r$-process elements we used model 1 by Cescutti et al. (2006) for both Ba and Eu. These empirical yields have been chosen to reproduce the surface abundances for $\mathrm{Ba}$ and $\mathrm{Eu}$ of low metallicity stars as well as the $\mathrm{Ba}$ and Eu solar abundances. Cescutti et al. (2006) have assumed that $\mathrm{Ba}$ is also produced as an $r$-process element in massive stars $\left(12-30 M_{\odot}\right)$, whereas Eu is considered to be a purely $r$-process element produced in the same range of masses.

For La we give new prescriptions following the same method as for $\mathrm{Ba}$ : we assume an $r$-process contribution in massive stars (12-30 $M_{\odot}$ ), besides the $s$-process contribution from low mass stars. The yields of this $r$-process contribution are summarized in Table 1, in which the mass fraction of newly produced La is given as function of the mass. The results for the solar neighborhood are shown in Sect. 5.

\section{Results for the solar vicinity}

We divide the $[\mathrm{Fe} / \mathrm{H}]$ axis in several bins and we compute the mean and the standard deviations from the mean of the ratios $[\mathrm{La} / \mathrm{Fe}]$ for all the data inside each bin. These results are shown in Table 2, where we also summarize the center and the dimension of each bin and the number of data points contained in each of them. In Fig. 2 we show the predictions of the chemical evolution model for La in the solar neighborhood using our prescriptions for the yields in massive stars and the prescriptions of Busso et al. (1999) for low mass stars, as described in the previous section. These results are new and the model well reproduces the trend of the stellar abundances at different $[\mathrm{Fe} / \mathrm{H}]$ and the solar abundance of lanthanum. We obtain a La mass fraction of $1.35 \times 10^{-9}$ very close to the solar value of Asplund et al. (2005) of $1.38 \times 10^{-9}$, as shown in Table 3 where the predicted and observed solar abundances are compared.

The yields adopted for $\alpha$ and iron peak elements in François et al. (2004) and for Ba and Eu in Cescutti et al. (2006) have been shown to well fit observational data in the solar neighborhood. In the next sections we check whether with the same nucleosynthesis prescriptions our model can explain the data in the other parts of the galactic disk.

\section{Abundance gradients compared with the $4 A L$ data}

We used the model described in Sect. 4 to predict the variation of the abundances of the studied elements along the galactic disk in the galactocentric range $5-17 \mathrm{kpc}$, at the present time. We then compared the abundances predicted by our model at the present time for all the elements with the observational data. To better understand the trend of the data, we divide the data in 6 bins as functions of the galactocentric distance. In each bin we compute the mean value and the standard deviations for all the elements. The results are shown in Table 4: in the first column we show the galactocentric distance range chosen for each bin, in the second column the mean galactocentric distance for the stars inside the

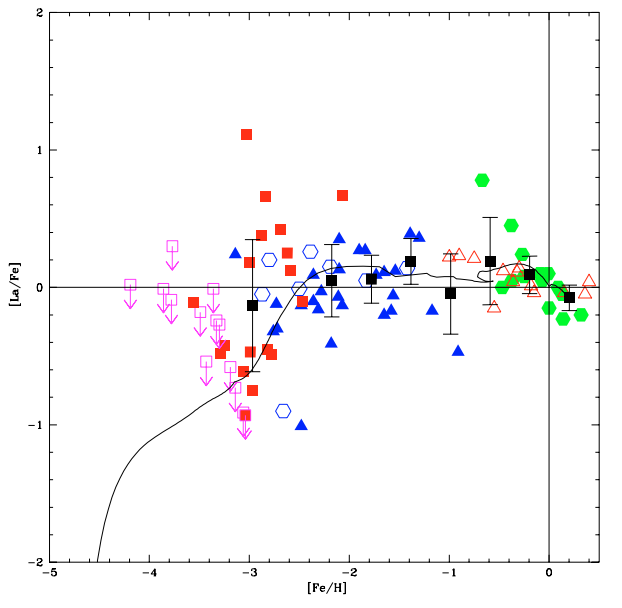

Fig. 2. $[\mathrm{La} / \mathrm{Fe}]$ versus $[\mathrm{Fe} / \mathrm{H}]$. The data are taken from François et al. (2006), (filled red squares, whereas the pink open squares are only upper limits), Cowan et al. (2005) (blue open hexagons), Venn et al. (2004) (blue solid triangles), Pompeia et al. (2003) (green filled hexagons) and McWilliam \& Rich (1994) (open red triangles). The black squares are the mean values of the data bins described in the Table 2. As error bars we consider the standard deviation (see Table 2). The solid line is the result of our model for La (see Table 1), normalized to the solar abundance as measured by Asplund et al. (2005).

considered bin, in the other columns the mean and the standard deviation computed for the abundances of every chemical element, inside the considered bin. For some stars it has not been possible to measure all the abundances. We plot the results of our model at the present day normalized to both the solar observed abundances by Asplund et al. (2005) and to the mean value of the abundance data by $4 \mathrm{AL}$ at the solar distance. For some elements there is a discrepancy between the predicted abundances at the present day by our model and the mean abundances of the observed Cepheids at the solar distance.

\section{1. $\alpha$-elements (O-Mg-Si-S-Ca)}

We plot the results for these elements in Fig. 3. There is a discrepancy between our predictions normalized to the solar abundances by Asplund et al. (2005) and the mean abundance of these elements for Cepheids at the solar galactocentric distance. The predictions of our model for these elements at the present time at $8 \mathrm{kpc}$ are supersolar, whereas the mean abundances of Cepheids for $\mathrm{Mg}, \mathrm{Ca}$ and $\mathrm{O}$ are subsolar and this difference is particularly evident for $\mathrm{Mg}$. This means that either our model predicts a too steep increase of metallicity in the last $4.5 \mathrm{Gyr}$ or that the absolute abundances of Cepheids are underestimated. Some uncertainties in the absolute abundances could exist, but the slope of the abundance distributions should not be affected. If these subsolar abundances were real, then one might think that they are the effect of some additional infall episode, occurring in the last 4.5 Gyr. However, the goal of this work is to reproduce the trend of the gradients, so here this problem can be neglected and we can compare the data with the model results normalized to the mean abundances of the Cepheids at $8 \mathrm{kpc}$. These results well reproduce the trend of the data for all the five elements. Moreover, in the case of Si we note that the data show very little spread (as the small standard deviation values indicate) and our model (the one normalized to the mean abundance at $8 \mathrm{kpc}$ ) perfectly lies over the mean value in each bin. For $S$ the values predicted by the model for small galactocentric distances are inside the error bar of the data but slightly low. The trend for $\mathrm{Ca}$ 
Table 2. The mean and the standard deviations for the abundance of $[\mathrm{La} / \mathrm{Fe}]$ for the stars inside each bin along the $[\mathrm{Fe} / \mathrm{H}]$ axis.

\begin{tabular}{ccccc}
\hline \hline bin center $[\mathrm{Fe} / \mathrm{H}]$ & bin dim. $[\mathrm{Fe} / \mathrm{H}]$ & mean $[\mathrm{La} / \mathrm{Fe}]$ & SD [La/Fe] & N. of data in the bin \\
\hline-2.97 & 1.20 & -0.13 & 0.48 & 29 \\
-2.17 & 0.40 & 0.04 & 0.26 & 15 \\
-1.78 & 0.40 & 0.06 & 0.17 & 7 \\
-1.38 & 0.40 & 0.18 & 0.17 & 5 \\
-0.99 & 0.40 & -0.04 & 0.29 & 4 \\
-0.59 & 0.40 & 0.19 & 0.32 & 5 \\
-0.19 & 0.40 & 0.09 & 0.14 & 13 \\
0.20 & 0.40 & -0.08 & 0.09 & 7 \\
\hline
\end{tabular}

Table 3. Element abundances by Asplund et al. (2005) in the present-day solar photosphere and in meteorites ( $\mathrm{C} 1$ chondrites) compared to the results of our model at the solar formation epoch.

\begin{tabular}{llccc|ccccc}
\hline \hline & Elem. & Photosphere & Meteorites & Model & & Elem. & Photosphere & Meteorites & Model \\
\hline 8 & $\mathrm{O}$ & $8.66 \pm 0.05$ & $8.39 \pm 0.02$ & 8.67 & 25 & $\mathrm{Mn}$ & $5.39 \pm 0.03$ & $5.47 \pm 0.03$ & 5.44 \\
12 & $\mathrm{Mg}$ & $7.53 \pm 0.09$ & $7.53 \pm 0.03$ & 7.57 & 26 & $\mathrm{Fe}$ & $7.45 \pm 0.05$ & $7.45 \pm 0.03$ & 7.41 \\
14 & $\mathrm{Si}$ & $7.51 \pm 0.04$ & $7.51 \pm 0.02$ & 7.58 & 27 & $\mathrm{Co}$ & $4.92 \pm 0.08$ & $4.86 \pm 0.03$ & 4.88 \\
16 & $\mathrm{~S}$ & $7.14 \pm 0.05$ & $7.16 \pm 0.04$ & 7.20 & 28 & $\mathrm{Ni}$ & $6.23 \pm 0.04$ & $6.19 \pm 0.03$ & 6.23 \\
20 & $\mathrm{Ca}$ & $6.31 \pm 0.04$ & $6.29 \pm 0.03$ & 6.25 & 29 & $\mathrm{Cu}$ & $4.21 \pm 0.04$ & $4.23 \pm 0.06$ & 4.13 \\
21 & $\mathrm{Sc}$ & $3.05 \pm 0.08$ & $3.04 \pm 0.04$ & 3.05 & 30 & $\mathrm{Zn}$ & $4.60 \pm 0.03$ & $4.61 \pm 0.04$ & 4.53 \\
22 & $\mathrm{Ti}$ & $4.90 \pm 0.06$ & $4.89 \pm 0.03$ & 4.90 & 56 & $\mathrm{Ba}$ & $2.17 \pm 0.07$ & $2.16 \pm 0.03$ & 2.19 \\
23 & $\mathrm{~V}$ & $4.00 \pm 0.02$ & $3.97 \pm 0.03$ & 3.59 & 57 & $\mathrm{La}$ & $1.13 \pm 0.05$ & $1.15 \pm 0.06$ & 1.11 \\
24 & $\mathrm{Cr}$ & $5.64 \pm 0.10$ & $5.63 \pm 0.05$ & 5.59 & 63 & $\mathrm{Eu}$ & $0.52 \pm 0.06$ & $0.49 \pm 0.04$ & 0.56 \\
\hline
\end{tabular}

Table 4. The mean value and the standard deviation inside each bin for $\mathrm{O}, \mathrm{Mg}, \mathrm{Si}$ and $\mathrm{S}$. The table for all the elements is available in the electronic Appendix.

\begin{tabular}{cccccccccc}
\hline \hline $\begin{array}{c}\text { Galactocentric } \\
\text { distance range }\end{array}$ & $\begin{array}{c}\text { Mean GC } \\
\text { distance }(\mathrm{kpc})\end{array}$ & $\begin{array}{c}\text { Mean } \\
{[\mathrm{O} / \mathrm{H}]}\end{array}$ & $\begin{array}{c}\text { SD } \\
{[\mathrm{O} / \mathrm{H}]}\end{array}$ & $\begin{array}{c}\text { Mean } \\
{[\mathrm{Mg} / \mathrm{H}]}\end{array}$ & $\begin{array}{c}\text { SD } \\
{[\mathrm{Mg} / \mathrm{H}]}\end{array}$ & $\begin{array}{c}\text { Mean } \\
{[\mathrm{Si} / \mathrm{H}]}\end{array}$ & $\begin{array}{c}\text { SD } \\
{[\mathrm{Si} / \mathrm{H}]}\end{array}$ & $\begin{array}{c}\text { mean } \\
{[\mathrm{S} / \mathrm{H}]}\end{array}$ & $\begin{array}{c}\text { SD } \\
{[\mathrm{S} / \mathrm{H}]}\end{array}$ \\
\hline$<6.5 \mathrm{kpc}$ & 5.76 & 0.16 & 0.17 & -0.19 & 0.17 & 0.21 & 0.13 & 0.37 & 0.19 \\
$6.5<-<7.5 \mathrm{kpc}$ & 7.10 & -0.08 & 0.13 & -0.19 & 0.10 & 0.07 & 0.06 & 0.17 & 0.08 \\
$7.5<-<8.5 \mathrm{kpc}$ & 8.00 & -0.06 & 0.13 & -0.19 & 0.13 & 0.06 & 0.06 & 0.09 & 0.10 \\
$8.5<-<9.5 \mathrm{kpc}$ & 8.96 & -0.12 & 0.16 & -0.21 & 0.11 & 0.04 & 0.04 & 0.08 & 0.17 \\
$9.5<-<11 \mathrm{kpc}$ & 10.09 & -0.16 & 0.19 & -0.22 & 0.18 & -0.07 & 0.07 & -0.11 & 0.20 \\
$>11 \mathrm{kpc}$ & 12.33 & -0.19 & 0.21 & -0.32 & 0.13 & -0.16 & 0.08 & -0.23 & 0.15 \\
\hline
\end{tabular}

is nicely followed by our model and the data for Ca show very little spread. On the other hand, the trend of $\mathrm{Mg}$ shows a shallower slope toward the galactic center than the other $\alpha$-elements. This is probably due to the lack of $\mathrm{Mg}$ data for the stars located from 4 to $6.5 \mathrm{kpc}$, which determine the steep slope for the other $\alpha$-elements. The results of the model, if we use the prescriptions for the halo gas density of model A by Chiappini et al. (2001) well reproduce the data up to $10 \mathrm{kpc}$ but model B better reproduces the data for greater galactocentric distances. For this reason in the next sections we will show only the results of model B. In Table 5 we show the slopes of the gradients for all the studied elements, as predicted by model B. The gradients become flatter towards the outermost disk regions, in agreement with the Cepheid data. Each element has a slightly different slope, due to the different production timescales and nucleosynthesis processes. In particular, $\alpha$-elements $(\mathrm{O}, \mathrm{Mg}, \mathrm{Si}, \mathrm{Ca}$ etc.) generally have flatter slopes than the Fe-peak-elements. In addition, there are differences even among the $\alpha$-elements such as $\mathrm{Si}$ and $\mathrm{Ca}$ relative to $\mathrm{Mg}$ and $\mathrm{O}$ : the slightly steeper slope of $\mathrm{Si}$ and $\mathrm{Ca}$ is due to the fact that these elements are produced also by type Ia $\mathrm{SNe}$, whereas $\mathrm{O}$ and $\mathrm{Mg}$ are not. In general, elements produced on longer timescales have steeper gradients. This is confirmed by the observations not only of Cepheids but also of open clusters and HII regions. The predicted gradients for $s$ - and $r$-process elements seem flatter than all the others. The reason for this is that they are produced in very restricted stellar mass ranges producing an increase of their abundances at low metallicities until they reach a constant value for $[\mathrm{Fe} / \mathrm{H}]>-3.0$ (see Fig. 2). The variations between gradients may be statistically significant, in particular those derived from Cepheids: all the Cepheids have similar atmospheric parameters (atmospheric temperature, surface gravity); their relative abundances are much less affected than the absolute abundances by the effect of using LTE models instead of recent NLTE, 3D models. Therefore the gradients from Cepheids seem to be established.

\subsection{Iron peak elements (Sc-Ti-Co-V-Fe-Ni-Zn-Cu-Mn-Cr)}

The ten elements of the so-called iron peak are plotted in Figs. 4 and 5. The present time predictions of our model for iron peak abundances are super-solar at $8 \mathrm{kpc}$, as for the $\alpha$-elements. On the other hand, the mean values for iron peak elements in Cepheids in the bin at $8 \mathrm{kpc}$ are in general solar, except $\mathrm{Zn}$, which is super-solar and Sc and Co, which are sub-solar. Nevertheless the model gives a prediction for the trends of the gradients for these elements which is very good, in particular in the cases of $\mathrm{V}, \mathrm{Fe}, \mathrm{Ni}, \mathrm{Mn}$ and $\mathrm{Cr}$, as shown by the results of the model normalized to the mean value of the bin centered at $8 \mathrm{kpc}$. 
Table 5. Model results for present time gradients for each element. We show the gradients computed as a single slope, for all the range of galactocentric distance considered, and as two slopes: from 4 to $14 \mathrm{kpc}$ and from 16 to $22 \mathrm{kpc}$.

\begin{tabular}{l|ccccccccc}
\hline \hline & $\mathrm{Fe}$ & $\mathrm{O}$ & $\mathrm{Mg}$ & $\mathrm{Si}$ & $\mathrm{S}$ & $\mathrm{Ca}$ & $\mathrm{Cu}$ & $\mathrm{Zn}$ & $\mathrm{Ni}$ \\
\hline$\frac{\Delta[\mathrm{el} / \mathrm{H}]}{\Delta R}(\mathrm{dex} / \mathrm{kpc})$ from 4 to $22 \mathrm{kpc}$ & -0.036 & -0.028 & -0.031 & -0.033 & -0.034 & -0.034 & -0.050 & -0.038 & -0.034 \\
$\frac{\Delta[\mathrm{el} / \mathrm{H}]}{\Delta R}(\mathrm{dex} / \mathrm{kpc})$ from 4 to $14 \mathrm{kpc}$ & -0.052 & -0.035 & -0.039 & -0.045 & -0.047 & -0.047 & -0.070 & -0.054 & -0.047 \\
$\frac{\Delta[\mathrm{el} / \mathrm{H}]}{\Delta R}(\mathrm{dex} / \mathrm{kpc})$ from 16 to $22 \mathrm{kpc}$ & -0.012 & -0.011 & -0.012 & -0.012 & -0.012 & -0.012 & -0.014 & -0.012 & -0.012 \\
\hline \hline & $\mathrm{Sc}$ & $\mathrm{Ti}$ & $\mathrm{V}$ & $\mathrm{Cr}$ & $\mathrm{Mn}$ & $\mathrm{Co}$ & $\mathrm{Ba}$ & $\mathrm{Eu}$ & $\mathrm{La}$ \\
\hline \hline$\frac{\Delta[\mathrm{el} / \mathrm{H}]}{\Delta R}(\mathrm{dex} / \mathrm{kpc})$ from 4 to $22 \mathrm{kpc}$ & -0.036 & -0.032 & -0.038 & -0.036 & -0.038 & -0.037 & -0.021 & -0.030 & -0.021 \\
$\frac{\Delta[\mathrm{lel} / \mathrm{H}]}{\Delta R}(\mathrm{dex} / \mathrm{kpc})$ from 4 to $14 \mathrm{kpc}$ & -0.051 & -0.043 & -0.056 & -0.052 & -0.057 & -0.055 & -0.032 & -0.036 & -0.032 \\
$\frac{\Delta[\mathrm{lel} / \mathrm{H}]}{\Delta R}(\mathrm{dex} / \mathrm{kpc})$ from 16 to $22 \mathrm{kpc}$ & -0.012 & -0.012 & -0.011 & -0.012 & -0.011 & -0.011 & -0.009 & -0.013 & -0.008 \\
\hline
\end{tabular}

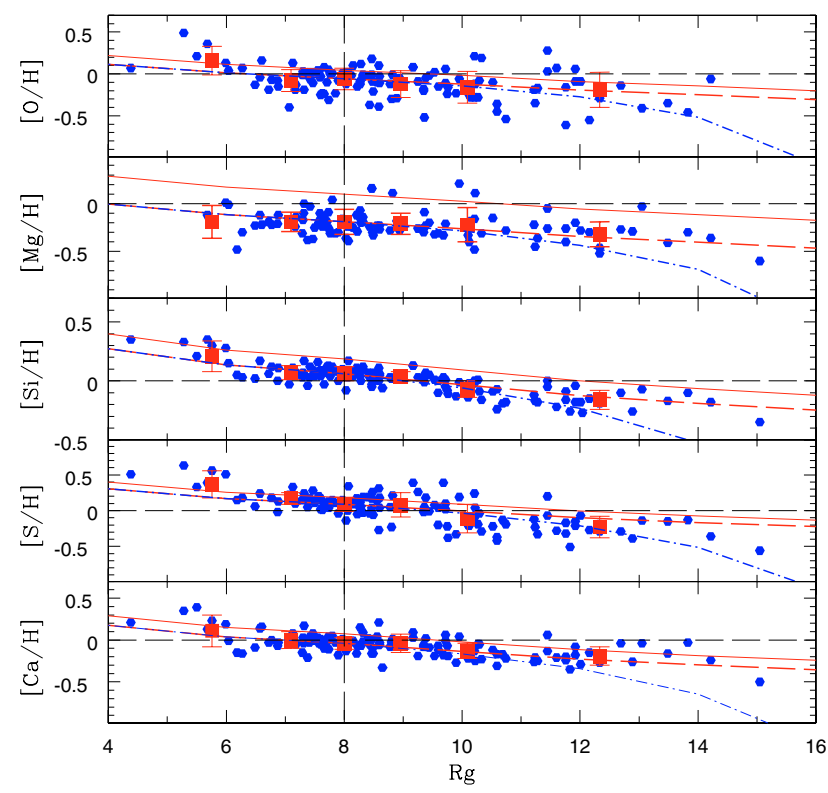

Fig. 3. Abundances for $\mathrm{O}, \mathrm{Mg}, \mathrm{Si}, \mathrm{S}$ and $\mathrm{Ca}$ as functions of the galactocentric distance. The blue dots are the data by $4 \mathrm{AL}$, the red squares are the mean values inside each bin only for the data by $4 \mathrm{AL}$ and the error bars are the standard deviations (see Table 4). The thin solid line is our model normalized to the observed solar abundances by Asplund et al. (2005), whereas the thick dashed line is normalized to the mean value at the bin centered in $8 \mathrm{kpc}$ (the galactocentric distance of the Sun). The dash-dotted line is the result of the model with the prescriptions for the halo gas density of model A by Chiappini et al. (2001) normalized to the mean value of the bin centered in $8 \mathrm{kpc}$ (cf. Sect. 3).

A problem is present for Co, for which a too low abundance is predicted by the model at galactocentric distances $>12 \mathrm{kpc}$.

\subsection{Neutron capture elements}

It is well known that these elements present a large spread at low metallicities, which is not yet understood (see Cescutti et al. 2006, and references therein). Since the Cepheids are young metal-rich stars, this problem is not important. As shown in Fig. 6, the spread in the data as a function of galactocentric distance is small. In the case of Eu our model well reproduces both the observed gradient and the mean value for the Cepheid abundance at $8 \mathrm{kpc}$. On the other hand, the mean value of the La abundance in the data by $4 \mathrm{AL}$ at the solar galactocentric distance is about a factor of 1.5 higher than the predicted abundance by our model and the predictions for La show slightly steeper gradients than the data. In Fig. 6, we show the predicted trend of the neutron capture element barium. For this element there are no data

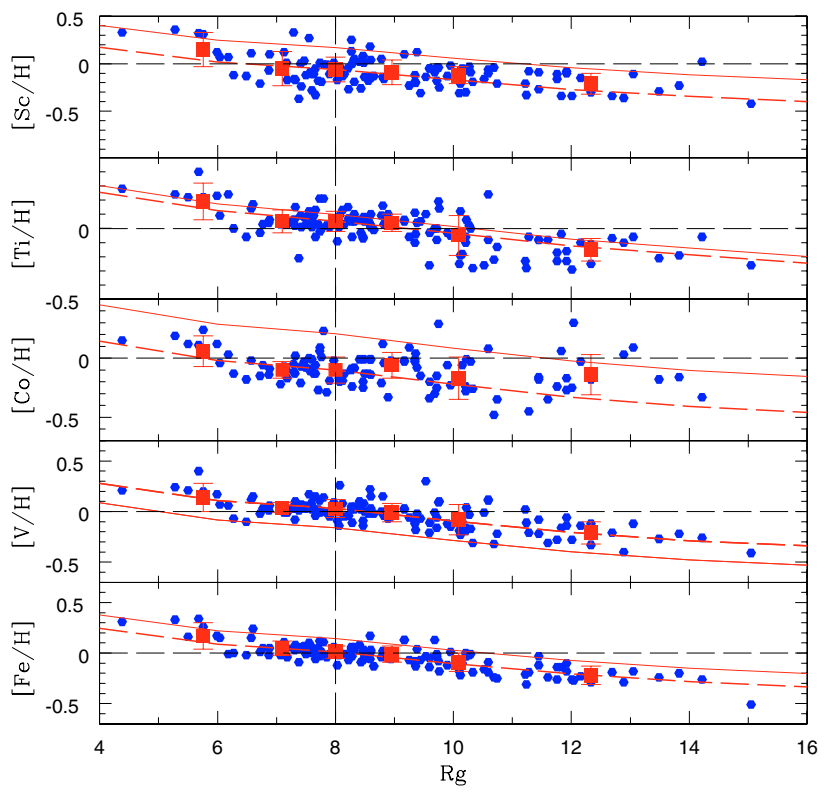

Fig. 4. Gradients for $[\mathrm{Sc} / \mathrm{H}],[\mathrm{Ti} / \mathrm{H}],[\mathrm{Co} / \mathrm{H}],[\mathrm{V} / \mathrm{H}]$ and $[\mathrm{Fe} / \mathrm{H}]$. The models and the symbols are the same as in Fig. 3.

by $4 \mathrm{AL}$; therefore, we just show our predictions, which have to be confirmed by future observations.

\section{Predicted abundance gradients compared with other sets of data}

We compare the results of our models with different sets of observational data, as described in Sect. 2. Only the data by Yong et al. (2006) refer to Cepheids. However, these data and 4AL data are not homogeneous because of the different way in which the abundances are derived. As a consequence of this, we apply the offsets calculated by Yong et al. (2006), on the basis of a representative sample of stars analyzed and measured by both authors, to compare the two sets of data. We compare the Cepheids and the Daflon \& Cunha (2004) data for OB stars with the model at the present time, normalized to the mean value at $8 \mathrm{kpc}$ for the data by 4AL, whereas we compare observational data of red giants and open clusters with the model to the solar formation time normalized at the observed solar abundances by Asplund et al. (2005).

\section{1. $\alpha$-elements (O-Mg-Si-S-Ca)}

In Fig. 7, we show the comparison for $\mathrm{O}, \mathrm{Mg}, \mathrm{Si}, \mathrm{S}$ and $\mathrm{Ca}$ data with our model. Although the observations are from completely 


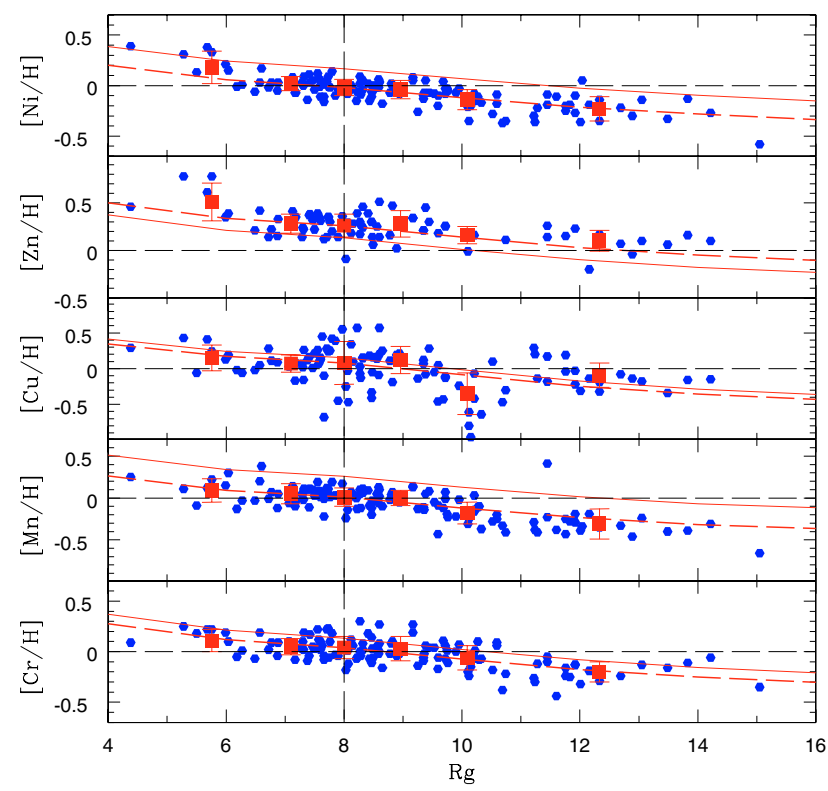

Fig. 5. Gradients for $[\mathrm{Ni} / \mathrm{H}],[\mathrm{Zn} / \mathrm{H}],[\mathrm{Cu} / \mathrm{H}],[\mathrm{Mn} / \mathrm{H}]$ and $[\mathrm{Cr} / \mathrm{H}]$. The models and the symbols are the same as in Fig. 3.

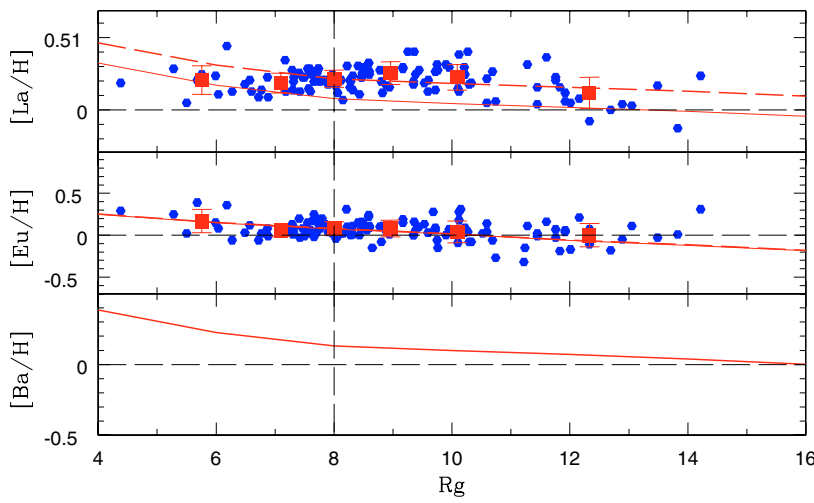

Fig. 6. Gradients for $[\mathrm{La} / \mathrm{H}],[\mathrm{Ba} / \mathrm{H}]$ and $[\mathrm{Eu} / \mathrm{H}]$. The models and the symbols are the same as in Fig. 3. Note that for Ba we show only the model predictions.

different types of astronomical objects (OB stars, red giants, open clusters and Cepheids), they are in agreement with each other and with our model. Nevertheless, the data by Yong et al. (2006) and the data by Daflon \& Cunha (2004) show a larger spread than the data by $4 \mathrm{AL}$, in particular for Ca. The data by Carraro et al. (2004) for the open cluster Saurer 1 at the galactocentric distance of $18.7 \mathrm{kpc}$ for all the considered elements, except $\mathrm{Mg}$, are slightly above the predictions of our model.

\subsection{The iron peak elements (Ti-Mn-Co-Ni-Fe)}

We show the iron peak elements in Fig. 8. The data by Yong et al. (2005) seem to have a gradient in agreement with our model if we take into account some possible offset in the data, as considered by Yong et al. (2006). In particular, the abundances of $\mathrm{Mn}$ in this data set are below our model predictions and the 4AL data. In the data by Yong et al. (2005), the open cluster Berkeley 31, which is at about $13 \mathrm{kpc}$, shows abundances lower than those predicted by our model and the set of data by $4 \mathrm{AL}$ for all the iron peak elements, with the exception of Co. On the other hand, the set of data by Carney et al. (2005) shows an almost flat trend and again lower abundances for the iron peak elements than the

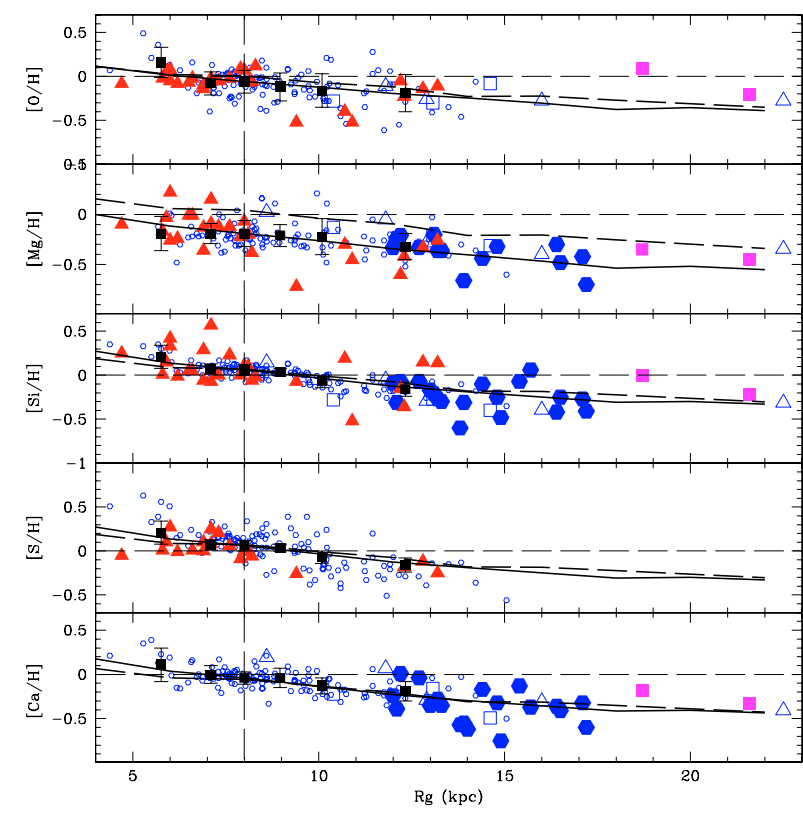

Fig. 7. The gradients for $\mathrm{O}, \mathrm{Mg}, \mathrm{Si}, \mathrm{S}$ and $\mathrm{Ca}$ compared with different sets of data. The small open circles are the data by $4 \mathrm{AL}$, the black squares are the mean values inside each bin for the data by $4 \mathrm{AL}$ and the error bars are the standard deviations (see table 4). The red solid triangles are the data by Daflon \& Cunha (2004) (OB stars), the open blue squares are the data by Carney et al. (2005) (red giants), the blue solid hexagons are the data by Yong et al. (2006) (Cepheids), the blue open triangles are the data by Yong et al. (2005) (open clusters) and the magenta solid squares are the data by Carraro et al. (2004) (open clusters). The most distant value for Carraro et al. (2004) and Yong et al. (2005) refers to the same object: the open cluster Berkeley 29. The thin solid line is our model at the present time normalized to the mean value of the bin centered at $8 \mathrm{kpc}$ for Cepheids stars by $4 \mathrm{AL}$; the dashed line represents the predictions of our model at the epoch of the formation of the solar system normalized to the observed solar abundances by Aspund et al. (2005). This prediction should be compared with the data for red giant stars and open clusters (Carraro et al. 2004; Carney et al. 2005; Yong et al. 2005).

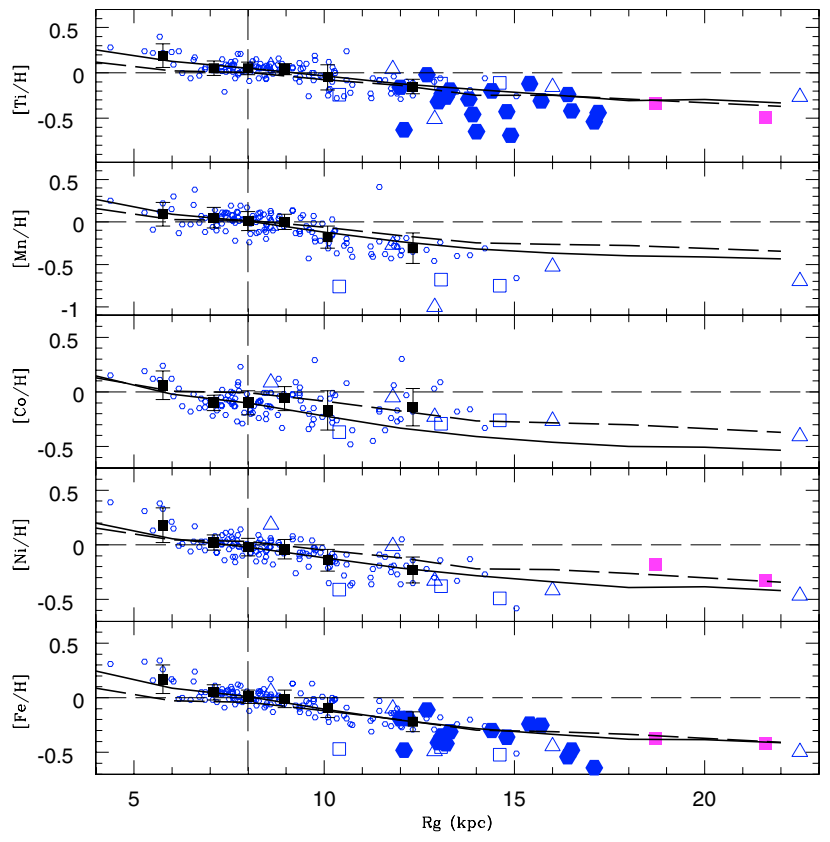

Fig. 8. Gradients for Ti, Mn, Co, Ni and Fe. The model and the symbols are the same as in Fig. 7. 


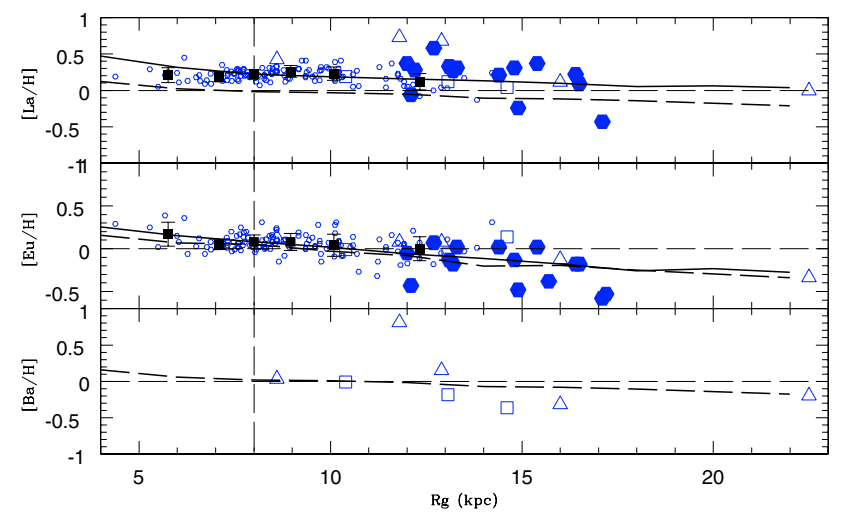

Fig. 9. Gradients for $\mathrm{La}, \mathrm{Eu}$ and $\mathrm{Ba}$. The model and the symbols are the same as in Fig. 7.

abundances of the data by 4AL and those predicted by the model. This is probably due to the fact that the data are from old and evolved objects, as giant stars are, with a not well estimated age. The data by Yong et al. (2006), that includes the abundances for $\mathrm{Ti}$ and $\mathrm{Fe}$, are in agreement with our model and the data by $4 \mathrm{AL}$, even if they seem to present slightly steeper gradients. The open cluster abundances as measured by Carraro et al. (2004) are in agreement with our model, in particular for Fe, while for $\mathrm{Ti}$ and $\mathrm{Ni}$ the model fits both open cluster abundances inside the error bars, which is about 0.2 dex.

\subsection{The neutron capture elements (La-Eu-Ba)}

We show the neutron capture elements in Fig. 9. The data for Eu are taken from the set of data by Yong et al. (2006, Cepheids), Yong et al. (2005, open clusters) and Carney et al. (2005, red giants), and they are in agreement with our model with the exception of a large spread in the data by Yong et al. (2006). Some problems arise for La. The trend of the gradients is similar for the sets of data but the absolute values of the La abundances in the sets of data by Yong et al. (2006), Yong et al. (2005) and Carney et al. (2005) are systematically lower than the ones of 4AL and, without the offset, it is impossible to make a comparison. Therefore, we apply an offset of +0.3 dex to all observational data (with the exception of the 4AL data) to better show all the sets of data. This can also be the consequence of the different way of calculating the abundances, as explained in Yong et al. (2006). The most important results are the slopes of the gradients rather than the absolute abundances. With this offset applied to the data, the two open clusters (Berkeley 31 and NGC 2141), measured by Yong et al. (2005), still present an abundance of La larger than the one predicted by our model and the mean abundance of the data by 4AL; finally, the data of Yong et al. (2006) again have a large spread. Nevertheless, the comparison is acceptable and the abundance of the most distant cluster is well fitted. The results for $\mathrm{Ba}$ are similar to those for $\mathrm{La}$. We have to apply an offset of +0.3 dex to the data by Carney et al. (2005), for the reasons explained above. The results are also quite good with the exceptions of the two open clusters mentioned before, which show a larger $\mathrm{Ba}$ abundance when compared to the results of our model. For both these open clusters there is only one measured star and it is possible that the stars chosen to be analyzed could be peculiar in terms of chemical abundances of $s$-process elements and so they should not be considered in the explanation of the gradients.
The data in the outer parts of disk are still insufficient to completely constrain our models. Moreover, the existing samples show scatter. Two factors can affect the abundance gradients in the outer part: observational uncertainties in both abundances and distance, and the fact that the outer parts could reflect a more complex chemical evolution. Moreover, there are some suggestions that the open clusters and giants in the outer part of the disk could have been accreted. However, despite these uncertainties, our chemical evolution model, where the halo density is assumed constant with radius out to $\sim 20 \mathrm{kpc}$, predicts abundances in agreement with those measured in the outer disk.

\section{Conclusions}

The aim of this work was to compare new observational data on the radial gradients for 17 chemical elements with the predictions of our chemical evolution model for the Milky Way. This model has been tested on the properties of the solar vicinity and contains a set of yields that best fits the abundances and abundance ratios in the solar vicinity, as shown in François et al. (2004).

The bulk of observational data comes from the abundances derived in a large number of Cepheids observed by 4AL. For the first time, it is possible to verify the predictions for many heavy elements with statistical validity.

The comparison between model predictions and observational data showed that our model well reproduces the gradients of almost all the elements that we analyzed. Since abundance gradients can impose strong constraints both on the mechanism of galaxy formation, in particular of the galactic disk, and the nucleosynthesis prescriptions, we can conclude that:

- The model for the Milky Way disk formation, assuming an inside-out building-up of the disk as suggested originally by Matteucci \& François (1989), can be considered successful; for almost all the considered elements, we find a good fit to the observational data ranging from 5 to $17 \mathrm{kpc}$. In particular, the model assuming a constant total surface mass density for the halo best fits the data of Cepheids. At large galactocentric distances the halo mass distribution influences the abundance gradients (see Chiappini et al. 2001).

- In our chemical evolution model we adopt a threshold in the gas density for star formation in the disk of $7 M_{\odot} \mathrm{pc}^{-2}$, whereas for the halo phase we have several options with and without a threshold. The threshold in the halo is $4 M_{\odot} \mathrm{pc}^{-2}$. We also assume a constant surface mass density for the halo or variable with galactocentric distance. This is important for the gradients at very large galactocentric distances, where the enrichment from the halo predominates over the enrichment occurring in the thin disk, thus influencing the abundances at such large distances.

We conclude that to reproduce the flat gradients suggested by the abundance measurements at large galactocentric distances, we need to assume a constant density distribution and a threshold in the star formation during the halo phase. However, there are still many uncertainties in the data at very large galactocentric distances and only more data will allow us to draw firm conclusions on this important point.

- The chosen nucleosynthesis prescriptions (empirical yields by François et al. 2004) successfully reproduce the abundance gradients of each specific element, as well as the $[\mathrm{el} / \mathrm{Fe}]$ vs. $[\mathrm{Fe} / \mathrm{H}]$ relations in the solar neighborhood, as shown already by François et al. (2004). 
- We also presented new results concerning the [La/Fe] vs. $[\mathrm{Fe} / \mathrm{H}]$ relation in the solar neighborhood. The data are the new ones by François et al. (2006). We conclude that La has the same origin as Ba: the bulk of La originates from low mass stars in the range $1-3 M_{\odot}$ as an $s$-process element, but a fraction of La originates, as an $r$-process element, from stars in the mass range $12-30 M_{\odot}$.

Acknowledgements. We thank Francesco Calura and Antonio Pipino for several useful comments. G.C. and F.M. acknowledge funds from MIUR, COFIN 2003, prot. No. 2003028039. C.C. acknowledges partial support by the INAF PRIN grant CRA 1.06.08.02.

\section{References}

Akerman, C. J., Carigi, L., Nissen, P. E., Pettini, M., \& Asplund, M. 2004, A\&A, 414,931

Alibés, A., Labay, J., \& Canal, R. 2001, A\&A, 370, 1103

Andrievsky, S. M., Bersier, D., Kovtyukh, V. V., et al. 2002a, A\&A, 384, 140

Andrievsky, S. M., Kovtyukh, V. V., Luck, R. E., et al. 2002b, A\&A, 381, 32

Andrievsky, S. M., Kovtyukh, V. V., Luck, R. E., et al. 2002c, A\&A, 392, 491

Andrievsky, S. M., Luck, R. E., Martin, P., et al. 2004, A\&A, 413, 159

Asplund, M., Grevesse, N., \& Sauval, A. J. 2005, ASPC, 336, 25A

Boisser, S., \& Prantzos, N. 1999, MNRAS, 307, 857

Bono, G., Marconi, M., Cassisi, S., et al. 2005, ApJ, 621, 966

Busso, M., Gallino, R., \& Wasserburg, G. J. 1999, ARA\&A, 37, 239

Busso, M., Gallino, R., Lambert, D. L., Travaglio, C., \& Smith, V. V. 2001, ApJ, 557,802

Cappellaro, E., Evans, R., \& Turatto, M. 1999, A\&A, 351, 459

Carney, B. W., Yong, D., Teixera de Almeida, M. L., \& Seitzer, P. 2005, AJ, 130, 1111

Carraro, G., Bresolin, F., Villanova, S., et al. 2004, AJ, 128, 1676
Chang, R. X., Hou, J. L., Shu, C. G., \& Fu, C. Q. 1999, A\&AS, 141, 491 Cescutti, G., François, P., Matteucci, F., Cayrel, R., \& Spite, M. 2006, A\&A, 448, 557

Chiappini, C., Matteucci, F., \& Gratton, R. G. 1997, ApJ, 477, 765

Chiappini, C., Matteucci, F., \& Romano, D. 2001, ApJ, 554, 1044

Chiappini, C., Romano, D., \& Matteucci, F. 2003a, MNRAS, 339, 63

Chiappini, C., Matteucci, F., \& Meynet, G. 2003b, A\&A, 410, 257

Cowan, J. J., Sneden, C., Beers, T. C., et al. 2005, ApJ, 627, 238

Daflon, S., Cunha, K. 2004, ApJ, 617, 1115

Feast, M. W., \& Walker, A. R. 1987, ARA\&A, 25, 345

François, P., Matteucci, F., Cayrel, R., et al. 2004, A\&A, 421, 613

François, P., Depagne, E., Hill, V., et al. 2006, in preparation

Greggio, L., \& Renzini, A. 1983, A\&A, 118, 217

Grevesse, N., \& Sauval, A. J. 1998, Space Sci. Rev., 85, 161

Henry, R. B. C., Edmunds, M. G., \& Köppen, J. 2000, ApJ, 541, 660

Hou, J. L., Prantzos, N., \& Boissier, S. 2000, A\&A, 362, 921

Kennicutt, R. C. Jr. 1989, ApJ, 344, 685

Kennicutt, R. C. Jr. 1998, ApJ, 498, 541

Liang, Y. C., Zhao, G., \& Shi, J. R. 2001, A\&A, 374, 936

Luck, R. E., Gieren, W. P., Andrievsky, S. M., et al. 2003, A\&A, 401, 939

Martin, C. L., \& Kennicutt, R. C. Jr. 2001, ApJ, 555, 301

Matteucci, F., \& François, P. 1989, MNRAS, 239, 885

Matteucci, F., \& Greggio, L. 1986, A\&A, 154, 279

McWilliam, A., \& Rich, R. M. 1994, ApJS, 91, 749

Pagel, B. E. J., \& Tautvaisiene, G. 1995, MNRAS, 276, 505

Pompeia, L., Barbuy, B., \& Grenon, M. 2003, ApJ, 592, 1173

Prantzos, N., \& Boisser, S. 2000, MNRAS, 313, 338

Reid, M. J. 1993, ARA\&A, 31, 345

Scalo, J. M. 1986, FCPh, 11, 1

van den Hoek, L. B., \& Groenewegen, M. A. T. 1997, A\&AS, 123, 305

Yong, D., Carney, B. W., \& Teixera de Almeida, M. L. 2005, AJ, 130, 597

Yong, D., Carney, B. W., Teixera de Almeida, M. L., \& Pohl, B. L. 2006, AJ, 131,2256

Woosley, S. E., \& Weaver, T. A. 1995, ApJ, 101, 181 
G. Cescutti et al.: Abundance gradients in the MW, Online Material $p 1$

\section{Online Material}


G. Cescutti et al.: Abundance gradients in the MW, Online Material p 2

Table 4. The mean value and the standard deviation inside each bin for $\mathrm{Ca}, \mathrm{Sc}, \mathrm{Ti}, \mathrm{V}, \mathrm{Cr}, \mathrm{Mn}, \mathrm{Fe}, \mathrm{Co}, \mathrm{Ni}, \mathrm{Cu}, \mathrm{Zn}, \mathrm{La}$ and $\mathrm{Eu}$.

\begin{tabular}{|c|c|c|c|c|c|c|c|c|c|c|c|}
\hline $\begin{array}{l}\text { galactocentric } \\
\text { distance range }\end{array}$ & $\begin{array}{c}\text { mean GC } \\
\text { distance }(\mathrm{kpc})\end{array}$ & $\begin{array}{c}\text { mean } \\
{[\mathrm{Ca} / \mathrm{H}]}\end{array}$ & $\begin{array}{c}\mathrm{SD} \\
{[\mathrm{Ca} / \mathrm{H}]}\end{array}$ & $\begin{array}{c}\text { mean } \\
{[\mathrm{Sc} / \mathrm{H}]}\end{array}$ & $\begin{array}{c}\mathrm{SD} \\
{[\mathrm{Sc} / \mathrm{H}]}\end{array}$ & $\begin{array}{l}\text { mean } \\
{[\mathrm{Ti} / \mathrm{H}]}\end{array}$ & $\begin{array}{c}\mathrm{SD} \\
{[\mathrm{Ti} / \mathrm{H}]}\end{array}$ & $\begin{array}{l}\text { mean } \\
{[\mathrm{V} / \mathrm{H}]}\end{array}$ & $\begin{array}{c}\mathrm{SD} \\
{[\mathrm{V} / \mathrm{H}]} \\
\end{array}$ & & \\
\hline$<6.5 \mathrm{kpc}$ & 5.76 & 0.11 & 0.19 & 0.15 & 0.18 & 0.19 & 0.13 & 0.14 & 0.14 & & \\
\hline $6.5<-<7.5 \mathrm{kpc}$ & 7.10 & 0.00 & 0.10 & -0.05 & 0.18 & 0.05 & 0.08 & 0.04 & 0.05 & & \\
\hline $7.5<-<8.5 \mathrm{kpc}$ & 8.00 & -0.04 & 0.07 & -0.06 & 0.13 & 0.05 & 0.07 & 0.03 & 0.09 & & \\
\hline $8.5<-<9.5 \mathrm{kpc}$ & 8.96 & -0.04 & 0.11 & -0.09 & 0.13 & 0.04 & 0.06 & -0.01 & 0.09 & & \\
\hline $9.5<-<11 \mathrm{kpc}$ & 10.09 & -0.13 & 0.09 & -0.12 & 0.09 & -0.05 & 0.14 & -0.08 & 0.15 & & \\
\hline$>11 \mathrm{kpc}$ & 12.33 & -0.19 & 0.11 & -0.21 & 0.11 & -0.15 & 0.08 & -0.21 & 0.11 & & \\
\hline $\begin{array}{l}\text { galactocentric } \\
\text { distance range }\end{array}$ & $\begin{array}{c}\text { mean GC } \\
\text { distance }(\mathrm{kpc})\end{array}$ & $\begin{array}{c}\text { mean } \\
{[\mathrm{Cr} / \mathrm{H}]}\end{array}$ & $\begin{array}{c}\mathrm{SD} \\
{[\mathrm{Cr} / \mathrm{H}]}\end{array}$ & $\begin{array}{c}\text { mean } \\
{[\mathrm{Mn} / \mathrm{H}]}\end{array}$ & $\begin{array}{c}\mathrm{SD} \\
{[\mathrm{Mn} / \mathrm{H}]}\end{array}$ & $\begin{array}{c}\text { mean } \\
{[\mathrm{Fe} / \mathrm{H}]}\end{array}$ & $\begin{array}{c}\mathrm{SD} \\
{[\mathrm{Fe} / \mathrm{H}]}\end{array}$ & $\begin{array}{c}\text { mean } \\
{[\mathrm{Co} / \mathrm{H}]}\end{array}$ & $\begin{array}{c}\mathrm{SD} \\
{[\mathrm{Co} / \mathrm{H}]}\end{array}$ & & \\
\hline$<6.5 \mathrm{kpc}$ & 5.76 & 0.11 & 0.11 & 0.09 & 0.14 & 0.17 & 0.13 & 0.06 & 0.13 & & \\
\hline $6.5<-<7.5 \mathrm{kpc}$ & 7.10 & 0.05 & 0.08 & 0.05 & 0.12 & 0.05 & 0.07 & -0.10 & 0.07 & & \\
\hline $7.5<-<8.5 \mathrm{kpc}$ & 8.00 & 0.04 & 0.11 & 0.01 & 0.11 & 0.01 & 0.06 & -0.10 & 0.11 & & \\
\hline $8.5<-<9.5 \mathrm{kpc}$ & 8.96 & 0.03 & 0.12 & 0.00 & 0.09 & -0.01 & 0.08 & -0.06 & 0.11 & & \\
\hline $9.5<-<11 \mathrm{kpc}$ & 10.09 & -0.06 & 0.12 & -0.18 & 0.13 & -0.09 & 0.09 & -0.17 & 0.18 & & \\
\hline$>11 \mathrm{kpc}$ & 12.33 & -0.20 & 0.10 & -0.31 & 0.18 & -0.22 & 0.09 & -0.14 & 0.17 & & \\
\hline $\begin{array}{l}\text { galactocentric } \\
\text { distance range }\end{array}$ & $\begin{array}{c}\text { mean GC } \\
\text { distance }(\mathrm{kpc})\end{array}$ & $\begin{array}{l}\text { mean } \\
{[\mathrm{Ni} / \mathrm{H}]}\end{array}$ & $\begin{array}{c}\mathrm{SD} \\
{[\mathrm{Ni} / \mathrm{H}]}\end{array}$ & $\begin{array}{c}\text { mean } \\
{[\mathrm{Cu} / \mathrm{H}]}\end{array}$ & $\begin{array}{c}\mathrm{SD} \\
{[\mathrm{Cu} / \mathrm{H}]}\end{array}$ & $\begin{array}{c}\text { mean } \\
{[\mathrm{Zn} / \mathrm{H}]}\end{array}$ & $\begin{array}{c}\mathrm{SD} \\
{[\mathrm{Zn} / \mathrm{H}]}\end{array}$ & $\begin{array}{c}\text { mean } \\
{[\mathrm{La} / \mathrm{H}]}\end{array}$ & $\begin{array}{c}\mathrm{SD} \\
{[\mathrm{La} / \mathrm{H}]}\end{array}$ & $\begin{array}{c}\text { mean } \\
{[\mathrm{Eu} / \mathrm{H}]}\end{array}$ & $\begin{array}{c}\mathrm{SD} \\
{[\mathrm{Eu} / \mathrm{H}]}\end{array}$ \\
\hline$<6.5 \mathrm{kpc}$ & 5.76 & 0.18 & 0.16 & 0.15 & 0.18 & 0.51 & 0.20 & 0.21 & 0.10 & 0.17 & 0.14 \\
\hline $6.5<-<7.5 \mathrm{kpc}$ & 7.10 & 0.02 & 0.07 & 0.07 & 0.12 & 0.28 & 0.10 & 0.19 & 0.07 & 0.05 & 0.06 \\
\hline $7.5<-<8.5 \mathrm{kpc}$ & 8.00 & -0.02 & 0.08 & 0.08 & 0.30 & 0.26 & 0.12 & 0.22 & 0.06 & 0.08 & 0.08 \\
\hline $8.5<-<9.5 \mathrm{kpc}$ & 8.96 & -0.04 & 0.09 & 0.12 & 0.19 & 0.28 & 0.14 & 0.26 & 0.08 & 0.08 & 0.10 \\
\hline $9.5<-<11 \mathrm{kpc}$ & 10.09 & -0.14 & 0.10 & -0.35 & 0.29 & 0.16 & 0.09 & 0.23 & 0.09 & 0.04 & 0.13 \\
\hline$>11 \mathrm{kpc}$ & 12.33 & -0.23 & 0.12 & -0.09 & 0.17 & 0.10 & 0.11 & 0.12 & 0.11 & 0.00 & 0.14 \\
\hline
\end{tabular}

